
Cahiers
de ea Recherche
Furles Droits
Fondamentaux
Cahiers de la recherche sur les droits fondamentaux

6 | 2008

Pouvoirs exceptionnels et droits fondamentaux

\title{
Le maire, " pivot » de la loi sur la prévention de la délinquance du 5 mars 2007
}

Jean-Manuel Larralde

\section{OpenEdition}

Édition électronique

URL : https://journals.openedition.org/crdf/6887

DOI : $10.4000 /$ crdf.6887

ISSN : 2264-1246

Éditeur

Presses universitaires de Caen

Édition imprimée

Date de publication : 31 décembre 2008

Pagination : 131-140

ISBN : 978-2-84133-259-5

ISSN : $1634-8842$

Référence électronique

Jean-Manuel Larralde, « Le maire, « pivot » de la loi sur la prévention de la délinquance du 5 mars

2007 », Cahiers de la recherche sur les droits fondamentaux [En ligne], 6 | 2008, mis en ligne le 11

décembre 2020, consulté le 15 novembre 2022. URL : http://journals.openedition.org/crdf/6887 ; DOI : https://doi.org/10.4000/crdf.6887 


\title{
Le maire, " pivot" de la loi sur la prévention de la délinquance du 5 mars 2007
}

\author{
Jean-Manuel LARRALDE \\ Professeur à I'Université de Caen Basse-Normandie
}

I. Le renforcement des moyens d'information du maire en matière de lutte contre la délinquance

A. Le renforcement des conseils locaux

B. La mise en place d'informations partagées

II. De nouveaux moyens d'action pour le maire

A. L' « accompagnement parental "

B. Le « rappel à l'ordre »

Dans un contexte social tout à fait particulier (celui des affrontements urbains du mois de novembre 2005 et des manifestations nationales contre le Contrat première embauche de février à avril 2006), les violences que l'on qualifie de "non crapuleuses ", c'est-à-dire non motivées par le vol, ont atteint des dimensions spectaculaires. La loi $\mathrm{n}^{\circ}$ 2007-297 du 5 mars 2007 sur la prévention de la délinquance entend donc apporter de nouvelles réponses aux problèmes posés par la lutte contre de nouvelles conduites délictueuses. Ce texte, complexe et hétérogène ${ }^{1}$, divisé en neuf chapitres ${ }^{2}$, repose sur deux idées directrices.

En premier lieu, la loi adopte volontairement une approche globalisante de la notion de « prévention » de la délinquance, qui s'appuie sur l'ensemble des mesures non pénales permettant d'empêcher la commission d'infractions ${ }^{3}$. Selon M. P. Houillon, rapporteur de la loi devant
l'Assemblée nationale, la prévention stricto sensu peut en effet revêtir trois volets différents : la prévention primaire qui constitue l'ensemble des moyens orientés vers la modification des conditions criminogènes de l'environnement physique et social global susceptible d'entraîner le passage à l'acte délinquant; la prévention secondaire qui se concentre sur l'identification et l'intervention préventive à l'égard de groupes ou de populations qui présentent des facteurs de risque de délinquance du fait de leur environnement social, de leurs difficultés scolaires... ; la prévention tertiaire qui concerne les actions pouvant être entreprises pour éviter la récidive et qui use de mesures individualisées à l'égard des délinquants. La notion de prévention selon la loi se caractérise donc par son caractère d'anticipation, en amont de toute infraction, elle permet d'agir sur les facteurs identifiés ou supposés de la délinquance.

1. Le texte, centré sur le traitement de la délinquance des mineurs, intègre également des mesures concernant les violences conjugales, le contrôle et l'euthanasie des chiens dangereux, les infractions sexuelles, la consommation de drogues, les troubles du voisinage, l'hospitalisation d'office de personnes atteintes de troubles mentaux, la fermeture des locaux contenant des produits inflammables ou dangereux... La loi précise également les compétences des gardes champêtres, qui sont placés sous l'autorité du maire de la commune sur le territoire de laquelle ils exercent leurs fonctions.

2. 1) dispositions générales; 2) dispositions de prévention fondées sur l'action sociale et éducative ; 3 ) dispositions tendant à limiter les atteintes aux biens et à prévenir les troubles de voisinage ; 4) dispositions fondées sur l'intégration ; 5) dispositions relatives à la prévention d'actes violents pour soi-même ou pour autrui ; 6) dispositions tendant à prévenir la toxicomanie et certaines pratiques addictives; 7) dispositions tendant à prévenir la délinquance des mineurs ; 8) dispositions organisant la sanction-réparation et le travail d'intérêt général ; 9) dispositions diverses.

3. Rapport fait par M. P. Houillon au nom de la commission des lois constitutionnelles, de la législation et de l'administration générale de la République sur le projet de loi adopté par le Sénat $\left(\mathrm{n}^{\circ}{ }^{3338}\right)$ relatif à la prévention de la délinquance, déposé le 15 novembre 2006. 
En second lieu, le texte entend explicitement faire du maire le pivot local de la lutte contre la délinquance. Cette idée part d'un postulat: en raison de sa proximité avec ses administrés, le maire serait le mieux placé pour exercer une mission de coordination des actions de prévention de la délinquance. Pour les rédacteurs de la loi, le maire «ne peut pas rester un simple spectateur. Il faut qu'il ait les moyens juridiques d'agir. Il ne s'agit pas de faire en sorte qu'il puisse se substituer à la police ou à la justice, mais de veiller à ce qu'il dispose des informations qu'il n'a pas $»^{4}$. Selon le premier alinéa de l'article $1^{\text {er }}$ de la loi du 5 mars 2007, le maire concourt par son pouvoir de police à l'exercice des missions de "prévention de la délinquance ", ce qui complète l'article L.2211-1 du Code général des collectivités territoriales (CGCT) qui prévoyait déjà que ce pouvoir de police « concourt à l'exercice des missions de sécurité publique ». Plus précisément encore, en vertu du nouvel article L.2211-4 CGCT, « le maire anime, sur le territoire de la commune, la politique de prévention de la délinquance et en coordonne la mise en œuvre ». À l'évidence, le rôle de proximité du maire est apparu tout à fait intéressant pour le législateur, grâce à l'étendue des compétences de la commune qui permet à l'exécutif de cette collectivité territoriale d'agir dans des domaines variés de la prévention, en prenant en compte des éléments de la politique sociale, éducative, urbanistique, voire médicale..., tout autant que des aspects de police administrative.

La loi opère donc un double mouvement au profit des maires des communes de France, en renforçant tout à la fois les informations dont ils disposent dans le cadre de la lutte contre la délinquance (I) et leurs moyens d'action, tant dans une perspective de prévention que de sanction (II).

\section{Le renforcement des moyens $d^{\prime}$ information du maire en matière de lutte contre la délinquance}

La désignation du maire comme autorité pilote de la lutte contre la délinquance est destinée à dépasser le foisonnement d'acteurs sur le terrain, analysée comme une source d'inefficacité et de brouillage des compétences et des responsabilités. Avec la loi du 5 mars 2007, le maire bénéficie d'un accroissement considérable des informations dont il peut disposer en matière de prévention (et d'action) contre la délinquance, grâce au renforcement des conseils locaux (A) et à la mise en place d'un système d'informations partagées (B).

\section{A. Le renforcement des conseils locaux}

Avec le décret no 83-459 du 8 juin 1983 sont apparus les Conseils communaux de prévention de la délinquance (CCPD), instances de concertation et de planification des programmes lancés principalement dans le cadre de la politique de la ville. La circulaire interministérielle du 28 octobre 1997 met ensuite en œuvre des Contrats locaux de sécurité (CLS) signés par le préfet, le procureur de la République et le maire, dont le but est de permettre la mobilisation de l'ensemble des acteurs concernés en mettant en œuvre des actions précises (prévention en milieu scolaire, aide aux victimes...). Concrétisant cette tendance, la loi $\mathrm{n}^{0}$ 2001-1062 du 15 novembre 2001 relative à la sécurité quotidienne dispose que «le maire est associé par le préfet à la définition des actions de prévention de la délinquance et de lutte contre l'insécurité ». En raison des confusions entre CLS et CCPD, le décret $n^{\circ}$ 2002-999 du 17 juillet 2002 remplace les CCPD par les Conseils locaux de sécurité et de prévention de la délinquance (CLSPD) qui jouent également le rôle de comité de suivi du CLS. Désormais, c'est le maire qui est au centre du dispositif puisqu'il préside cette instance qu'il est donc chargé d'animer. Le CLSPD est composé du préfet et de représentants de services de l'État, du procureur de la République, le cas échéant du président de l'Établissement public de coopération intercommunale (EPCI) compétent en matière de dispositifs locaux de prévention contre la délinquance, de représentants d'associations, établissements ou organismes œuvrant dans divers domaines : prévention, sécurité, aide aux victimes, logement, transports collectifs, action sociale ou activités économiques ${ }^{5}$. En ce qui concerne ses compétences, le CLSPD est une structure de concertation, qui permet une définition collective des priorités de la lutte contre la délinquance. Ce conseil présente l'indéniable avantage de regrouper l'ensemble des acteurs concernés par la politique de prévention de la délinquance, mais également d'avoir un pilote identifié : le maire qui le préside. Ce dernier doit notamment dresser un constat des actions de prévention, définir les objectifs et actions coordonnés, et en suivre l'exécution.

Avec la loi du 7 mars 2007 (article L.2211-4 CGCT), les CLSPD sont généralisés puisqu'ils sont rendus obligatoires dans les communes de plus de 10000 habitants $^{6}$, ou dans un cadre intercommunal lorsqu'un EPCI à fiscalité propre « exerce la compétence relative aux dispositifs locaux de prévention de la délinquance ${ }^{7}$. On peut se demander quelle sera la portée exacte de cette généralisation (qui ne concernera qu'environ 350 communes nouvelles), car nombre de conseils déjà existants ne se réunissent en assemblée plénière qu'une ou deux fois par an,

4. Rapport de M. P. Houillon du 15 novembre 2006, précité.

5. $\mathrm{Au} 1^{\text {er }}$ mai 2006, 871 CLSPD avaient été conclus, représentant 25 millions d'habitants. Parmi ces CLSPD, 783 avaient été effectivement mis en place, alors qu'environ 250 CCPD étaient encore actifs en 2002. $40 \%$ de ces CLSPD sont intercommunaux (560 communaux et 311 intercommunaux).

6. Avec des dispositions spécifiques pour la ville de Paris. Voir l'article L.2512-13-1 CGCT.

7. Cette compétence est obligatoire pour les communautés urbaines (article L.5215-20 CGCT) et les communautés d'agglomération (article L.5216-5 CGCT). La loi précise, dans un but de simplification, que dans les communes membres d'un EPCI qui dispose d'un Conseil intercommunal de protection et de lutte contre la délinquance (CISPD), cette création est seulement facultative (article L.2211-4 deuxième alinéa CGCT). En outre, lorsqu'il existe un CISPD, c'est logiquement au président de l'EPCI que revient la mission d'animer et de coordonner les actions de prévention de la délinquance. Cependant, cette compétence doit se faire « sous réserve du pouvoir de police des maires des communes membres». 
ce qui ne permet pas de réel échange d'informations, ni de véritables actions coordonnées. Ces structures restent tributaires de la bonne volonté de leurs membres et l'on constate que seuls les CLSPD dotés d'une formation restreinte, de groupes de travail ou d'une cellule de veille (soit environ un tiers de ces conseils) ont une réelle efficacité opérationnelle.

L'article 9 de la loi crée par ailleurs une nouvelle structure dans les communes de plus de 10000 habitants: le Conseil pour les droits et les devoirs des familles (CDDF; article L.141-1 et L.141-2 nouveaux du Code de l'action sociale et des familles). Le législateur a voulu par cette création conférer une base légale à de nombreuses expériences menées localement, comme celle «des maisons des parents » ouvertes dans de nombreuses communes. Présidé par le maire et composé d'élus locaux, de représentants de l'État et « des personnes œuvrant dans les domaines de l'action sociale, sanitaire et éducative, de l'insertion et de la prévention de la délinquance ${ }^{8}$, ce conseil est présenté comme un lieu d'échange avec les familles en difficulté afin de les aider à trouver des solutions concrètes aux problèmes qu'elles rencontrent. Chaque conseil doit également définir son mode d'intervention auprès des familles, dans le respect de la libre administration des collectivités locales.

Le Conseil pour les droits et les devoirs des familles est une instance uniquement consultative, qui ne possède aucun pouvoir décisionnel, et a fortiori, aucun pouvoir de sanction. Il est seulement conçu comme un lieu de proposition et d'échanges entre ses membres et des familles connaissant des difficultés dans l'éducation de leurs enfants. En effet, contrairement au Conseil local de sécurité et de prévention de la délinquance, le Conseil pour les droits et les devoirs des familles s'intéresse à des situations individuelles, et non aux orientations générales de la politique de prévention de la délinquance 9 . Ses missions sont diverses: entendre les familles en difficulté et leur proposer des recommandations afin de prévenir les comportements de leurs enfants susceptibles soit de mettre en danger les enfants eux-mêmes, soit de porter atteinte à l'ordre public ; proposer au maire de saisir le président du Conseil général en vue de mettre en œuvre " une mesure d'accompagnement en économie sociale et familiale» lorsque «la situation d'une famille ou d'un foyer est de nature à compromettre l'éducation des enfants »; proposer un accompagnement parental, qui consiste en des actions de sou- tien et de suivi à la fonction éducative (article L.141-2 du Code de l'action sociale et des familles). Les parents qui s'estiment eux-mêmes dépassés par le comportement de leurs enfants pourront également demander au maire de leur commune à bénéficier d'un tel accompagnement.

La loi apparaît particulièrement floue en ce qui concerne les garanties offertes aux familles ou aux «foyers » (ces deux entités n'y étant d'ailleurs pas définies) qui devront venir s'expliquer devant le conseil. Le texte n'apporte, en effet, aucune précision sur le droit à la défense des familles mises en cause et sur la garantie d'une procédure objective et contradictoire. En outre, toute comparution devant le CDDF risque de stigmatiser un peu plus aux yeux de leur voisinage des familles en difficulté car, en pratique, le passage devant cette structure pourra difficilement rester confidentiel ${ }^{10}$. La Commission nationale Informatique et Libertés, dans son avis du 13 juin 2006, s'est montrée tout à fait critique, en regrettant que «se trouve ainsi institué un dispositif de signalement des mineurs et des familles à problèmes résidant dans la commune, sans qu'aucune garantie ne soit apportée ni sur l'origine des informations qui seraient utilisées pour procéder à ce signalement, ni sur les critères déclenchant ce signalement, ni sur les modalités de transmission et de traitement des informations et la nécessaire confidentialité de celles-ci ».

Présent à la tête de plusieurs conseils, le maire va désormais pouvoir bénéficier d'informations qui jusque-là lui étaient inaccessibles, car réservées aux professionnels de terrain. La loi du 5 mars 2007 rompt avec cet important élément de secret professionnel, en mettant en place des « informations partagées».

\section{B. La mise en place d'informations partagées}

La loi sur la prévention de la délinquance permet désormais d'étoffer l'information du maire sur l'absentéisme scolaire, ce qui complète les compétences dont le maire dispose déjà en matière de contrôle de l'obligation scolaire $^{11}$. En vertu de l'article L.131-1 du Code de l'éducation, l'instruction est obligatoire pour les enfants des deux sexes, français et étrangers, entre six et seize ans. L'article 12 de la loi du 5 mars $2007^{12}$ autorise désormais les maires à mettre en place des traitements automatisés contenant de nombreuses informations en provenance des Caisses d'allocation familiales ${ }^{13}$ et de l'inspecteur

8. Une relative liberté est laissée à chaque commune dans l'organisation du conseil : le maire peut présider lui-même le conseil ou le faire présider par un représentant, sans qu'il soit précisé que celui-ci soit un élu du Conseil municipal. Sa composition sera fixée librement dans chaque commune.

9. C'est d'ailleurs la raison pour laquelle les informations échangées dans ce cadre ont un caractère confidentiel, dont la divulgation peut entraîner les sanctions prévues à l'article 226-13 du Code pénal.

10. Même si le nouvel article L.141-1 du Code de l'action sociale et des familles prévoit que les informations communiquées aux membres du conseil «ne peuvent être divulguées à des tiers sous peine des sanctions prévues à l'article 226-13 du Code pénal ».

11. Voir notamment le décret $\mathrm{n}^{\circ}$ 2004-162 du 19 février 2004 portant modification du décret $\mathrm{n}^{\circ}$ 66-104 du 18 février 1966 relatif au contrôle de la fréquentation et de l'assiduité scolaires et aux sanctions que comportent, au regard du versement des prestations familiales et en matière pénale, les manquements à l'obligation scolaire et du Code pénal.

12. Nouveaux articles L.121-1, L.131-6, L.131-8, L.131-10 et L.214-13 du Code de l'éducation.

13. En application des articles L.552-4 et L.552-5 du Code de la sécurité sociale, le versement des prestations familiales est en effet subordonné à la production d'un certificat de scolarité, d'un certificat délivré par le préfet attestant que l'instruction est donnée dans la famille ou d'un certificat médical attestant que l'enfant ne peut pas être scolarisé pour des raisons de santé. De cette façon, les Caisses d'allocations familiales peuvent déceler des situations de non-scolarisation dont la connaissance pourrait être utile au maire. 
d'académie et recensant les enfants soumis à l'obligation scolaire dans chaque commune. Aujourd'hui, en effet, le recensement est uniquement annuel et dépend entièrement d'une démarche volontaire des parents qui doivent soit inscrire leur enfant auprès de la mairie, soit adresser au maire une déclaration d'instruction dans la famille. Afin de vérifier ces déclarations, le maire ne dispose actuellement que d'un seul moyen : effectuer un recoupement avec la liste des enfants inscrits dans les établissements de sa commune, transmise chaque mois par chacun d'entre $\operatorname{eux}^{14}$. L'idée retenue par la loi est que l'absentéisme scolaire constituant le signe avant-coureur de difficultés familiales d'ordre plus général, il est utile que le maire puisse obtenir des informations le plus rapidement possible pour pouvoir agir efficacement. Dans son avis du 13 juin 2006 sur le projet de loi, la Commission nationale Informatique et Libertés a d'ailleurs relevé « que la constitution par le maire d'un traitement automatisé de données à caractère personnel afin de recenser les enfants résidant dans sa commune soumis à l'obligation scolaire et d'améliorer le suivi de l'obligation d'assiduité scolaire s'inscrit dans le cadre des compétences qui lui sont reconnues par les articles L.131-6 et R.131-3 du Code de l'éducation. [...] Le projet de loi a donc pour objet l'enrichissement de cette liste par des données provenant des caisses d'allocations familiales et des inspections académiques ».

Il n'en reste pas moins que les maires pourront désormais disposer d'un ensemble d'informations extrêmement large et probablement excessif: identité de l'ensemble des enfants en âge scolaire résidant sur le territoire de la commune et donnant droit à l'ouverture de prestations familiales, dont le versement est conditionné à la production d'un certificat de scolarité ; avertissement prononcé par l'inspecteur d'académie à l'encontre d'un élève qui a manqué la classe sans motif légitime plus de quatre demi-journées dans le mois (article L.131-8 du Code de l'éducation); données transmises par le directeur de l'école ou le chef d'établissement en cas d'exclusion temporaire ou définitive ou en cas d'abandon en cours d'année scolaire ; avertissement...

Les sources d'information du maire plus directement en lien avec la délinquance sont également étoffées. Avec la loi n ${ }^{\circ}$ 2004-204 du 9 mars 2004 portant adaptation de la justice aux évolutions de la criminalité, les maires étaient déjà tenus informés sans délai par les responsables locaux de la police ou de la gendarmerie des infractions causant un trouble grave à l'ordre public commises sur le territoire de leur commune. Lors de la discussion du projet de loi sur la prévention de la délinquance, le Sénat a souhaité qu'ils soient désormais informés de l'ensemble des infractions (problèmes de voisinage ou désordres dans un quartier spécifique de la commune par exemple) causant un trouble à l'ordre public sur le territoire de leur commune, et non plus uniquement de celles considérées comme "graves». L'application effective de cette nouvelle mesure dépendra toutefois de l'implication concrète des procureurs de la République concernés ${ }^{15}$.

L'accroissement des informations à disposition du maire passe également par la reconnaissance de son rôle central dans le nouveau dispositif de secret professionnel en matière de prévention de la délinquance. Pour la loi, l'efficacité de l'action sociale sur le terrain ne dépend pas tant d'un manque d'intervenants qualifiés que d'un défaut de coordination de leur action et d'une carence dans l'utilisation de l'information existante ${ }^{16}$. Le travail en réseau dans ce domaine est rendu difficile dans la mesure où la plupart des professionnels sont tenus à une obligation de secret ou de confidentialité, dont la violation constitue un délit, en application de l'article 226-13 du Code pénal ${ }^{17}$. Désormais, lorsqu'une personne ou une famille fait l'objet de plusieurs interventions, l'article 8 de la loi (nouvel article L.121-6-2 du Code de l'action sociale et des familles) autorise les acteurs de l'action sociale à partager entre eux les informations (protégées par le secret professionnel ou non) dont ils disposent sur une personne ou une famille qui connaît une "aggravation de ses difficultés sociales, éducatives ou matérielles ${ }^{18}$. La loi ne fait ici que conférer une consécration législative à une pratique déjà courante sur le terrain, consistant à organiser des réunions de synthèse sur des situations individuelles.

Afin que les actions menées par différents intervenants soient efficaces et les informations utilisées au mieux, le maire, saisi par un professionnel, ou par le président du Conseil général, ou de sa propre initiative, nomme un coor-

14. Au cas par cas, les personnes autorisées à consulter la liste communale (à savoir les conseillers municipaux, les délégués départementaux de l'Éducation nationale, les assistantes sociales, les personnels d'enseignement, les agents de l'autorité, les inspecteurs d'académie et le directeur des services départementaux de l'Éducation nationale) peuvent en outre signaler au maire des omissions.

15. L'article 40-2 du Code de procédure pénale fait obligation au procureur d'aviser le maire des poursuites judiciaires données à la suite de leur signalement.

16. La loi a été ici indéniablement influencée par plusieurs faits divers qui ont bouleversé l'opinion publique, tels que la mort en août 2003 d'un petit garçon de neuf ans dans le quartier de Hautepierre, à Strasbourg, après avoir été torturé par ses parents, son oncle et sa grand-mère maternelle. Neuf travailleurs sociaux étaient déjà intervenus sur la situation de cet enfant et de sa famille...

17. Les assistants sociaux, quelle que soit la structure à laquelle ils appartiennent, sont tenus au secret professionnel (article L.411-2 du Code de l'action sociale et des familles), tout comme les personnels des centres communaux d'action sociale (article L.133-5). Le secret professionnel est également applicable à « toute personne participant aux missions du service de l'aide sociale à l'enfance» (article L.221-6). La loi prévoit également une obligation de secret professionnel pour les agents du service d'accueil téléphonique du service de la protection maternelle et infantile du Conseil général (article L.226-9). Par ailleurs, tous les intervenants dans le domaine de l'action sociale qui ont un statut de fonctionnaire de l'une des trois fonctions publiques sont astreints, même s'ils ne relèvent pas du secret professionnel, à une obligation « de discrétion professionnelle pour tous les faits, informations ou documents dont ils ont connaissance dans l'exercice ou à l'occasion de l'exercice de leurs fonctions » (article 26 de la loi n ${ }^{\circ} 83-634$ du 13 juillet 1983 portant droits et obligations des fonctionnaires).

18. La loi du 7 mars 2007 s’inspire ici de la technique du « secret partagé » mise en place par la loi n² 2002-303 du 4 mars 2002 relative aux droits du malade et à la qualité du système de santé : l'article L.1110-4 du Code de la santé publique autorise le partage d'informations médicales relatives à une même personne entre plusieurs professionnels de santé. 
donnateur ${ }^{19}$ qui a un double rôle: l'animation de l'équipe de travailleurs sociaux pour optimiser leurs différentes interventions, et l'information des personnes susceptibles de prendre les mesures adaptées, à savoir le maire et le président du Conseil général. Le caractère systématique de cette nomination a fait l'objet de nombreuses critiques tant de la part de l'Association des maires de France que des travailleurs sociaux ${ }^{20}$. La loi prévoit en outre une possibilité pour le coordonnateur de transmettre aux élus, s'il le juge utile, des informations «strictement nécessaires à l'accomplissement de la mission d'action sociale». Cette transmission ne constitue donc pas un droit pour le maire ou le président du Conseil général. En effet, les travailleurs sociaux ne divulgueront qu'au coordonnateur les informations à caractère confidentiel, celui-ci appréciera ensuite souverainement si les informations échangées entre professionnels doivent être transmises. En outre, le maire ne peut disposer de signalements à caractère confidentiel que dans ses domaines de compétence, c'est-à-dire ceux dans lesquels il est à même de proposer une intervention. A priori, aucun renseignement sur la situation individuelle d'une personne ou d'une famille ne devrait être transmis directement au maire. En pratique, certains rapports de force existants sur le terrain risquent de rendre délicat tout refus de transmissions d'informations au maire.

Ce système complexe de secret professionnel partagé s'avérera en outre délicat à mettre en place parce qu'il est sensiblement différent dans ses modalités pratiques et dans les garanties offertes aux professionnels et aux familles de celui adopté dans le cadre de la loi n ${ }^{0}$ 2007-293 du 5 mars 2007 relative à la protection de l'enfance ${ }^{21}$. On peut craindre que l'existence de deux mécanismes différents n'entraîne une confusion chez les professionnels et n'entrave l'efficacité des deux systèmes de partage des informations.

D’une manière plus générale, on peut s'interroger sur le bien-fondé du partage des informations les plus sensibles, qui ne devrait relever que des travailleurs sociaux en charge des familles concernées dans le cadre d'un projet social $^{22}$. Si le Code pénal protège de façon si forte le secret professionnel, c'est parce qu'il constitue une garantie fondamentale pour des personnes, souvent placées en situa- tion de vulnérabilité (malade face à son médecin, famille en difficulté face à une assistante sociale...), et des professionnels. Dans son avis du 21 septembre 2006, la Commission nationale consultative des droits de l'homme a d'ailleurs reconnu que le partage d'informations est nécessaire entre professionnels pour la mise en place de politiques ou de projets concertés, tout en mentionnant les « garanties qui doivent encadrer le partage de toute donnée sensible sur la vie privée et elle rappelle à cette occasion la nécessité de veiller à ce que, à tout le moins, les intéressés formalisent leur accord et soient informés des destinataires des informations». Ces garanties pourraient notamment s'inspirer de la circulaire conjointe du ministère de la Justice et du ministère de la Santé du 21 juin 1996 sur le secret partagé qui prévoit qu'il convient « de ne transmettre que les éléments nécessaires, de s'assurer que l'usager concerné est d'accord pour cette transmission ou tout au moins qu'il en a été informé ainsi que des éventuelles conséquences que pourra avoir cette transmission d'informations et de s'assurer que les personnes à qui cette transmission est faite sont soumises au secret professionnel et ont vraiment besoin, dans l'intérêt de l'usager, de ces informations ». Une transmission de données confidentielles insuffisamment encadrée risque de rendre plus délicat le rapport de confiance qui doit exister entre les personnes prises en charge et les travailleurs sociaux de terrain $^{23}$.

La mise en place de ce véritable « réseau » d'information autour de la figure du maire n'est pas une fin en soi. Elle constitue le socle indispensable aux nouveaux moyens d'action à destination des enfants et de leurs familles dont il est doté.

\section{De nouveaux moyens d'action pour le maire}

Détenteur d'informations essentielles, le maire peut désormais agir de plusieurs manières, ce qui entraîne un certain brouillage des catégories juridiques. En effet, le titulaire du pouvoir exécutif communal devient tout à la fois un véritable acteur du droit de la famille grâce à ses

19. Le quatrième alinéa de l'article (introduit par le Sénat lors de la discussion parlementaire) précise que lorsque les professionnels concernés relèvent tous de l'autorité du président du Conseil général (dans la pratique $80 \%$ des travailleurs sociaux dépendent du département) il reviendra au président du Conseil général de proposer le nom d'un coordonnateur que le maire désignera officiellement comme tel. Le président du Conseil général peut, en outre, désigner un coordonnateur en cas d'inaction du maire.

20. En outre, la question de l'identité du professionnel pouvant être choisi par le maire a été le point le plus controversé au Sénat. En effet, certains sénateurs ont craint que ce pouvoir n'entraîne un empiétement du maire sur les compétences du Conseil général en matière d'action sociale.

21. En vertu du nouvel article L.226-2-2 du Code de l'action sociale et des familles, "par exception à l'article 226-13 du Code pénal, les personnes soumises au secret professionnel qui mettent en œuvre la politique de protection de l'enfance définie à l'article L.112-3 ou qui lui apportent leur concours sont autorisées à partager entre elles des informations à caractère secret afin d'évaluer une situation individuelle, de déterminer et de mettre en œuvre les actions de protection et d'aide dont les mineurs et leur famille peuvent bénéficier. Le partage des informations relatives à une situation individuelle est strictement limité à ce qui est nécessaire à l'accomplissement de la mission de protection de l'enfance. Le père, la mère, toute autre personne exerçant l'autorité parentale, le tuteur, l'enfant en fonction de son âge et de sa maturité sont préalablement informés, selon des modalités adaptées, sauf si cette information est contraire à l'intérêt de l'enfant».

22. Et ce même si le Conseil constitutionnel a estimé dans sa décision n ${ }^{\circ} 2007-553$ DC du 3 mars 2007 que le dispositif de partage des informations présente des limitations et des précautions aptes à protéger la vie privée des intéressés. Note J.-E. Schoettl, La Semaine juridique (édition administration et collectivités territoriales), $\mathrm{n}^{\circ} 12,19$ mars 2007, 2071, p. 39 sq.

23. Selon H. Heurtebize, éducateur spécialisé et membre du Collectif national unitaire qui s'est constitué contre le projet de loi, «la réalité, c’est que l'on va pénétrer dans la vie privée des gens sans aucune garantie de confidentialité. À terme, cela bannit toute confiance entre l'éducateur et la personne prise en charge ». L. Mouloud, «Les maires enrôlés par Sarkozy », L’Humanité, 13 septembre 2006. 
possibilités d' «accompagnement parental», qui le font participer aux missions parentales et éducatives (A) et le premier maillon d'un dispositif répressif par le biais du « rappel à l'ordre» $(\mathrm{B})$.

\section{A. L'« accompagnement parental»}

En vertu du nouvel article L.141-2 du Code de l'action sociale et des familles, le maire "lorsqu'il ressort de ses constatations ou d'informations portées à sa connaissance que l'ordre, la sécurité ou la tranquillité publics sont menacés à raison du défaut de surveillance ou d'assiduité scolaire d'un mineur [...] peut proposer aux parents ou au représentant légal du mineur concerné un accompagnement parental». Cet accompagnement parental « consiste en un suivi individualisé au travers d'actions de conseil et de soutien à la fonction éducative » (article L.141-2, alinéa 2). Cette mesure fait l'objet d'une information de l'inspecteur d'académie, du chef d'établissement d'enseignement, du directeur de l'organisme débiteur des prestations familiales et du préfet. Au terme de l'accompagnement, est délivrée aux parents ou au représentant légal du mineur « une attestation comportant leur engagement solennel à se conformer aux obligations liées à l'exercice de l'autorité parentale ». En cas de refus sans motif légitime de l'accompagnement par les parents ou le représentant légal du mineur, le maire doit saisir le président du Conseil général en vue de la conclusion éventuelle d'un contrat de responsabilité parentale. Le contrat de responsabilité parentale (CRP), créé par la loi no 2006 396 du 31 mars 2006 pour l'égalité des chances ${ }^{24}$, a pour fonction de rappeler leurs obligations aux titulaires de l'autorité parentale. Proposé par le président du Conseil général, il comporte toute mesure d'aide et d'actions sociales de nature à remédier à la situation.

On ne peut ici que s'interroger sur la manière dont l'accompagnement parental pourra s'articuler avec ce contrat de responsabilité parentale ${ }^{25}$. Dans les deux cas, en effet, les mesures concrètes susceptibles d'être proposées aux familles sont similaires : suivi individualisé, aide à domicile, participation à des actions collectives d'aide à la parentalité, médiation familiale... De même, les situations susceptibles de conduire à proposer un accompagnement parental sont très proches de celles prévues pour la mise en place d'un CRP. En effet, si le premier peut être envisagé en cas de défaut de surveillance des parents ou de manquement à l'assiduité scolaire, le second peut l'être dans des hypothèses similaires, puisque l'article L.222-4-1 du Code de l'action sociale et des familles l'envisage « en cas d'absentéisme scolaire [...], de trouble porté au fonctionnement d'un établissement d'enseignement ou de tout autre difficulté liée à une carence de l'autorité parentale ». La seule différence entre les deux dispositifs semble être liée à l'existence ou non de troubles à la sécurité ou à la tranquillité publique: si le défaut de surveillance ou le manquement à l'assiduité scolaire de l'enfant provoquent des troubles à l'ordre public, le maire peut proposer un accompagnement parental. Ce dispositif semble faire double emploi avec le CRP, mais - et on peut penser qu'il s'agit là de la véritable intention du législateur - il pourrait constituer une sorte de première étape avant la conclusion d'un CRP, sans que la nécessité de cet échelon supplémentaire apparaisse vraiment justifiée.

La participation du maire aux missions parentales se manifeste, en outre, par une possibilité d'action indirecte, en matière de contrôle de l'utilisation des prestations familiales. Depuis la loi du 31 mars 2006 pour l'égalité des chances (nouvel article L.222-4-1 du Code de l'action sociale et des familles), le président du Conseil général peut, lorsque les parents ne respectent pas les obligations leur incombant en vertu du contrat de responsabilité parentale ou lorsqu'ils refusent de le signer sans motif légitime, demander au directeur de l'organisme débiteur des prestations familiales la suspension du versement de tout ou partie des prestations afférentes à l'enfant pour trois mois renouvelables jusqu'à un an, ou saisir le juge des enfants aux fins d'une mise sous tutelle des prestations familiales ${ }^{26}$. L'article 20 de la loi $n^{0}$ 2007-293 du 5 mars 2007 réformant la protection de l'enfance a modifié cette procédure, en transférant, du Code de la sécurité sociale au Code civil, les dispositions relatives à la tutelle aux prestations familiales, sous le titre «Mesure judiciaire d'aide à la gestion du budget familial». Certes, la loi sur la prévention de la délinquance ne permet au maire ni de suspendre, ni même de mettre sous tutelle les allocations familiales de familles d'enfants délinquants : l'article R.167-2 du Code de la sécurité sociale, qui fixe la liste de personnes qui peuvent demander au juge des enfants l'ouverture de la tutelle aux prestations familiales, ne mentionne toujours pas le maire ${ }^{27}$. Le projet de loi, qui prévoyait un article L.552-7 du Code de la sécurité sociale lui accordant ce pouvoir de saisine, n'a pas été retenu dans le texte final. Mais cette absence n'est guère surprenante, car une telle disposition se serait avérée redondante. En effet, en vertu du nouvel article 375-9-2 du Code

24. Voir le commentaire de ce contrat par J. Rochfeld, "Chronique de législation française », Revue trimestrielle de droit civil, avril-juin 2006 , p. 395 sq.

25. Étant entendu qu'une famille ne peut faire l'objet des deux mesures simultanément: en vertu de l'article L.141-2 du Code de l'action sociale et des familles, le maire doit vérifier, avant de proposer aux parents un accompagnement parental, qu'aucun contrat de responsabilité parentale n'a déjà été conclu avec eux. Le maire doit, en outre, tenir informé le Conseil général des mesures prises.

26. Dans les conditions prévues par l'article L.552-6 du Code de la sécurité sociale: «Dans le cadre de la mesure judiciaire d'aide à la gestion du budget familial prévue à l'article 375-9-1 du Code civil, le juge peut décider qu’une personne qualifiée, dite "délégué aux prestations familiales", perçoit tout ou partie des prestations familiales dues au bénéficiaire de la mesure ».

27. Les personnes habilitées à saisir le juge des enfants étant le père, la mère ou la personne investie du droit de garde sur le mineur au profit duquel est versée la prestation; la personne qui a la charge effective et permanente du mineur; les préfets; les organismes ou services débiteurs des prestations sociales; le directeur régional des affaires sanitaires et sociales; le chef du service régional de l'inspection du travail, de l'emploi et de la politique sociale agricoles; le directeur départemental des affaires sanitaires et sociales; le procureur de la République. Enfin, le juge des enfants peut d’office ouvrir la tutelle. Toute personne, autorité, organisme ou service, autre que le directeur départemental des affaires sanitaires et sociales, qui prend l'initiative de saisir le juge des enfants doit en informer immédiatement le directeur départemental qui fait connaître son avis au juge compétent. 
civil issu de la loi du 5 mars 2007 relative à la protection de l'enfance, «le maire ou son représentant au sein du Conseil pour les droits et devoirs des familles peut saisir le juge des enfants, conjointement avec l'organisme débiteur des prestations familiales, pour lui signaler, en application de l'article 375-9-1, les difficultés d'une famille». Cette vague mention des « difficultés d'une famille» renvoie en réalité à une situation beaucoup plus précise. En effet, l'article 3759-1 du Code civil prévoit que « lorsque les prestations familiales ne sont pas employées pour les besoins liés au logement, à l'entretien, à la santé et à l'éducation des enfants et que l'accompagnement en économie sociale et familiale prévu à l'article L.222-3 du Code de l'action sociale et des familles n'apparaît pas suffisant, le juge des enfants peut ordonner qu'elles soient, en tout ou partie, versées à une personne physique ou morale qualifiée, dite "délégué aux prestations familiales" ». Il s'agit donc d'une possibilité indirecte pour le maire de demander au juge, pour une durée d'un maximum de deux ans, la mise sous tutelle des allocations familiales. L'intervention du maire apparaît ici tout à fait ambiguë, car une telle mise sous tutelle des prestations sociales de l'enfant délinquant ne relève à l'évidence plus de la prévention de la délinquance, mais bien d'une démarche de sanction. Plus encore, une telle mesure risque de pénaliser davantage des familles marginalisées et en proie à de graves difficultés financières.

Ces deux nouvelles techniques apportent des arguments supplémentaires à ceux qui dénoncent la transformation progressive de la notion d'autorité parentale. Définie par l'article 371-1 premier alinéa du Code civil comme « un ensemble de droits et de devoirs ayant pour finalité l'intérêt de l'enfant », elle tend aujourd'hui à intégrer de très nombreux éléments de protection de la société et de l'ordre public. Ceci servant par ailleurs à justifier que cette même autorité parentale accorde de plus en plus de place aux autorités publiques pour l'évaluation de sa mise en œuvre ${ }^{28}$. Les exigences de protection de l'ordre public constituent également le fondement de la nouvelle procédure de « rappel à l'ordre » des auteurs d'incivilités, compétence désormais reconnue par la loi au maire de chaque commune française.

\section{B. Le « rappel à l'ordre »}

En vertu du nouvel article L.2212-2-1 CGCT, «lorsque des faits sont susceptibles de porter atteinte au bon ordre, à la sûreté, à la sécurité ou à la salubrité publiques, le maire ou son représentant peut procéder verbalement à l'endroit de leur auteur au rappel des dispositions qui s'imposent à celui-ci pour se conformer à l'ordre et à la tranquillité publics, le cas échéant en le convoquant en mairie ».
Cette procédure de « rappel à l'ordre » permet au maire de jouer un rôle que l'on pourrait qualifier de médiateur dans la lutte contre ce que l'on appelle désormais les « incivilités ", c'est-à-dire des faits qui ne sont pas tous susceptibles d'entraîner une qualification pénale (tels des manquements réitérés à la discipline par un élève d'un établissement scolaire). L'idée semble ici de favoriser une gestion non pénale de certains actes de délinquance compte tenu de l'autorité morale dont dispose le maire dans sa commune. Le texte ne prévoit qu'un simple rappel à l'ordre, ce qui signifie qu'il n'autorise nullement d'autres interventions du maire, comme par exemple une incitation à la réparation amiable d'un dommage. Il ne s'agit cependant pas d'une procédure anodine, ce qui explique qu'elle ait été précisément encadrée par le législateur. Ainsi, le rappel à l'ordre ne peut pas être effectué par un agent de la commune, mais seulement par le maire ou l'un des élus municipaux bénéficiant d'une délégation. En outre, si toute personne peut faire l'objet d'un rappel à l'ordre, le deuxième alinéa de l'article L.2212-2-1 CGCT précise que «le rappel à l'ordre d'un mineur intervient, sauf impossibilité, en présence de ses parents, de ses représentants légaux ou, à défaut, d'une personne exerçant une responsabilité éducative à l'égard de ce mineur ». Enfin, dans tous les cas, le rappel à l'ordre doit être formulé « verbalement». Il ne doit donc pas se matérialiser par un écrit remis à l'auteur des faits. Il ne s'agit que d'un face-à-face entre le maire et la personne concernée ${ }^{29}$. Mais le choix d'utiliser ce dispositif ne fait évidemment pas obstacle à la mise en œuvre de l'action publique s'il y a lieu.

La nouvelle procédure de l'article L.2212-2-1 CGCT s'insère dans les compétences de police administrative du maire (la loi parlant d'actes susceptibles de porter atteinte $\mathrm{au}$ «bon ordre, à la sûreté, à la sécurité ou à la salubrité publiques ») et elle lui permettra avant tout de rappeler les dispositions qui s'appliquent lorsqu'une personne a enfreint un de ses arrêtés de police municipale, ou toute mesure de police administrative ${ }^{30}$, ou encore lorsqu' elle a commis un acte contraventionnel de nature à porter atteinte à l'une des composantes de l'ordre public, comme, par exemple, la divagation d'animaux dangereux (article R.622-2 Code pénal), les bruits ou tapages injurieux ou nocturnes (article R.623-2), les violences légères (article R.6241), les destructions, dégradations et détériorations ayant entraîné un dommage léger (article R.635-1), l'abandon d'épaves, d'ordures, de déchets (article R.635-8), etc. Mais le domaine administratif n'est pas le seul champ d'application de cette procédure, car le maire pourra également formuler des rappels à l'ordre concernant des faits pouvant entraîner une peine contraventionnelle. L'utilisation de cette procédure en cas de délit ou de crime n'est en effet pas possible, car l'article 40 du Code de procédure pénale (rappelé à l'article 2211-2 CGCT) fait obligation au maire,

28. Voir, sur ce point, J. Rochfeld, «Chronique de législation française », Revue trimestrielle de droit civil, avril-juin 2007, p. 408.

29. On peut d'ailleurs s'interroger sur l'exacte portée normative du rappel à l'ordre, dès lors que celui-ci n'est que verbal, donc non formalisé, et qu'il n'emporte aucune autre conséquence, notamment en termes de sanction.

30. L'actualité récente a bien montré que le pouvoir de police administrative du maire était amené à s'exercer dans de nombreux domaines relevant de la lutte contre la délinquance : arrêtés antimendicité, interdiction de la circulation nocturne de certains mineurs, interdiction de la vente d'alcool... Pour un panorama général, voir G. Chavrier, Les Communes et la délinquance, Paris, Éditions du Moniteur, 2003. 
comme à toute autorité publique, de signaler au procureur de la République les crimes et délits dont il a connaissance dans l'exercice de ses fonctions. La possibilité d'un rappel à l'ordre pour des faits relevant de peines contraventionnelles sera toutefois très délicate à combiner avec celle de nature juridictionnelle déjà prévue par l'article 41-1 du Code de procédure pénale, qui permet au procureur (ou à l'un de ses délégués), de «procéder au rappel auprès de l'auteur des faits des obligations résultant de la loi », plutôt que de déclencher des poursuites, «s'il lui apparaît qu'une telle mesure est susceptible d'assurer la réparation du dommage causé à la victime, de mettre fin au trouble résultant de l'infraction ou de contribuer au reclassement de l'auteur des faits ». Cet «avertissement » concerne généralement des infractions de faible gravité, commises pour la première fois et n'est pas inscrit au casier judiciaire. Il sera certainement délicat pour les personnes concernées de distinguer entre ce rappel à l'ordre «juridictionnel » du procureur et le rappel à l'ordre «non juridictionnel » reconnu au maire... Lors de l'élaboration de la loi, certains maires ${ }^{31}$ comme certains procureurs de la République ${ }^{32}$ se sont montrés assez circonspects à l'égard des possibilités de rappel à l'ordre du maire, car ils voient dans cette pratique les prémices d'une sanction que l'autorité judiciaire est seule compétente à appliquer.

En outre, le nouvel article L.2212-2-1 CGCT ne fait qu'officialiser une pratique largement répandue: de nombreux maires avaient déjà recours à des admonestations verbales, justifiées par leur autorité morale auprès de leurs administrés, surtout dans les zones rurales et dans les petites villes ${ }^{33}$. Cette pratique a pu d'ailleurs être utile dans un grand nombre de cas, en permettant un rappel à l'ordre dès la constatation du comportement fautif. L'autorité immédiate du maire apparaît parfois plus efficace que celle du juge qui intervient évidemment toujours en décalage par rapport au fait générateur. Les maires procédant déjà à des rappels à l'ordre, en dehors de toute base juridique, on peut se demander s'il était vraiment utile de formaliser cette technique dans un texte législatif. La formalisation d'un dispositif souple risque, en effet, de rendre encore plus évidentes les disparités entre les différentes communes françaises et à contraindre les maires à mettre en place une procédure qui jusqu'alors relevait de leur pouvoir d'appréciation souveraine.

La loi du 5 mars 2007 constitue au final un texte complexe, mais surtout révélateur de certains errements actuels du législateur français et excessivement ambigu quant à ses finalités réelles.

En premier lieu, la loi sur la prévention de la délinquance ne semble guère s'être préoccupée de l'environnement législatif préexistant ou concomitant. La mise en cohérence des institutions, techniques et procédures envisagées par la loi sur l'égalité des chances de 2006 et des deux lois du 5 mars 2007, l'une sur la protection de l'enfance et l'autre sur la prévention de la délinquance, apparaît ainsi tout à fait problématique ${ }^{34}$. Dans le même sens, l'affirmation d'une place centrale pour le maire dans les domaines éducatifs et de la famille est assez douteuse au regard de l'architecture administrative française: depuis les lois de décentralisation de 1982-1983, renforcées sur ce point par la loi $n^{0}$ 2004-809 du 13 août 2004, la coordination de l'action sociale relève des départements, l'action sociale n'étant qu'une compétence facultative des communes ${ }^{35}$. À une période où le discours officiel est celui de la simplification du droit, la loi du 5 mars 2007 sur la prévention de la délinquance offre un fort mauvais exemple...

En second lieu - et surtout - ce texte législatif est très ambigu, en cherchant trop souvent à masquer ses véritables enjeux : les principales mesures contenues dans cette loi dite de "prévention » de la délinquance renvoient en réalité à des mesures à finalité répressive ${ }^{36}$. Comme l'indiquait en 2006 M. N. Sarkozy, alors ministre d'État, de l'Intérieur et de l'Aménagement du territoire, «la société a le devoir de sanctionner pour prévenir. Parce que la meilleure des préventions, c'est la sanction ${ }^{37}$. Depuis 2002, plusieurs textes législatifs répressifs se sont en effet accu-

31. L'association des petites villes de France a rappelé « qu'au-delà des pouvoirs de police qui leur sont aujourd'hui reconnus par les textes et qu'ils appliquent sans défaillance, leur mission relève avant tout de la médiation et non de la sanction. Il convient donc de ne pas mélanger les rôles de l'élu, du juge judiciaire et de la police». Cité par N. Demiati, «La prévention de la délinquance : un instrument du pouvoir local; Comment surveiller les "familles issues de l'immigration" pour mieux gouverner les villes de banlieues?», 27 février 2007, source: http://oumma.com/La-prevention-de-la-delinquanceun, 2366.

32. Voir, sur ce point, l'avis $\mathrm{n}^{\circ} 3434$ présenté par M. J.-M. Dubernard à l'Assemblée nationale le 14 novembre 2006 au nom de la commission des affaires culturelles, familiales et sociales sur le projet de loi adopté par le Sénat, relatif à la prévention de la délinquance.

33. Comme l'indique le sénateur J.-R. Lecerf en des termes encore plus explicites, une grande majorité des maires « ont déjà recours à cette pratique qui consiste, pour parler familièrement, “à remonter les bretelles” en cas d'incivilités ou de petites dégradations ». Rapport n 476 (2005-2006), précité.

34. Voir sur ce point J.-P. Rosenczveig, «La loi “prévention de la délinquance” », Recueil Dalloz, 2007, p. 640 sq.

35. L'article L.121-1 du Code de l'action sociale et des familles dispose que «le département définit et met en œuvre la politique d'action sociale, en tenant compte des compétences confiées par la loi à l'État, aux autres collectivités territoriales ainsi qu'aux organismes de sécurité sociale» et qu’il «coordonne les actions menées sur son territoire qui y concourent». La rédaction de l'article 5 du projet de loi sur la prévention de la délinquance a d'ailleurs été amendée à juste titre par le Sénat pour tenir compte de la compétence attribuée au Conseil général en matière de coordination et de pilotage de la politique d'action sociale. Le nouveau texte de l'article L.2211-4 du CGCT dispose cependant que l'action du maire doit s'accomplir « sous réserve des pouvoirs de l'autorité judiciaire et dans le respect des compétences du représentant de l'État, des compétences d'action sociale confiées au département et des compétences des collectivités publiques, des établissements et des organismes intéressés ». La référence spécifique aux départements a été ajoutée par le Sénat afin de rassurer certains présidents de Conseils généraux qui craignaient que les nouveaux pouvoirs reconnus aux maires en matière de prévention de la délinquance ne les conduise à interférer dans la politique d'action sociale des départements.

36. En dehors des éléments précédemment développés, la loi procède à une aggravation des sanctions pénales en matières de violences volontaires, d'entrave à la circulation des trains et d'usage de stupéfiants, à la légalisation des indicateurs en matière d'infractions sexuelles, à la légalisation de la provocation policière en matière de stupéfiants, au durcissement de la sanction en cas de récidive et des conditions permettant la réhabilitation pénale, à l'extension des pouvoirs des contrôleurs des sociétés de transports en matière de contrôles d'identité et de "rétentions ", etc.

37. Interview à la radio Europe 1 du lundi 30 octobre 2006. 
mulés ${ }^{38}$ et la loi sur la prévention de la délinquance participe à ces enjeux, sans le dire toujours explicitement, en comportant de très nombreux éléments de sanction. L’idée est ici que la sanction serait « une composante essentielle de la prévention grâce à ses vertus à la fois dissuasives et pédagogiques », car « la diversification de la réponse pénale permet [...] de développer, à côté de la sanction pure et simple, une dimension éducative importante ${ }^{39}$. La Commission nationale consultative des droits de l'homme s'était d'ailleurs expressément interrogée, dans son avis du 21 septembre 2006, «sur la philosophie d'un texte dont les finalités ne sont pas véritablement affichées et qui, mal- gré le titre annonçant un projet de loi sur la prévention de la délinquance, traite essentiellement de mesures de répression ou de moyens permettant de la mettre en œuvre ${ }^{40}$. On peut effectivement considérer que la loi renvoie à une logique de surveillance généralisée et de contrôle social de la population ${ }^{41}$, en favorisant la multiplication des fichiers, et en faisant de certains individus issus de milieux en difficulté des suspects en puissance ${ }^{42}$, ce qui constitue tout de même une perspective assez particulière de la prévention nécessaire en matière de lutte contre la délinquance.

38. La lutte contre l'insécurité étant désormais une thématique fort prisée du législateur français. Voir - principalement - les lois $n^{\circ} 95-73$ du 21 janvier 1995, loi d'orientation et de programmation pour la sécurité; n $\mathrm{n}^{\circ}$ 2002-1094 du 29 août 2002, loi d'orientation et de programmation pour la sécurité intérieure; $\mathrm{n}^{\circ}$ 2002-1138 du 9 septembre 2002, loi d'orientation et de programmation pour la justice; nº 2003-239 du 18 mars 2003 , loi pour la sécurité intérieure; $\mathrm{n}^{\circ}$ 2003-1119 du 26 novembre 2003, loi relative à la maîtrise de l'immigration, au séjour des étrangers en France et à la nationalité ; $\mathrm{n}^{\circ}$ 2004204 du 9 mars 2004 portant adaptation de la justice aux évolutions de la criminalité; nº 2006-64 du 23 janvier 2006, loi relative à la lutte contre le terrorisme et portant dispositions diverses relatives à la sécurité et aux contrôles frontaliers; $\mathrm{n}^{\circ}$ 2006-911, du 24 juillet 2006, loi relative à l'immigration et à l'intégration...

39. Rapport de M. J.-R. Lecerf, précité.

40. La Commission avait d'ailleurs rappelé à cette occasion son précédent avis du 14 novembre 2002 dans lequel elle réaffirmait que «la sécurité ne s'oppose pas aux libertés, notamment le respect de la dignité humaine, la liberté d'aller et venir, les droits de la défense, sans lesquelles il n'est pas de véritable sécurité».

41. Ce qui rappelle évidemment les célèbres analyses de Michel Foucault développées dans son ouvrage Surveiller et punir; naissance de la prison, Paris, Gallimard (Bibliothèque des histoires), 1975.

42. Voir l'intervention de M. J.-P. Michel lors de l'audition des ministres devant la commission des lois du Sénat réunie le mardi 5 septembre 2006 sous la présidence de M. J.-J. Hyest. Avis n 477 (2005-2006) de M. N. About, fait au nom de la commission des affaires sociales, déposé le 6 septembre 2006. 